\title{
Preparation and Analysis of Porous Oxychloride Titanium Oxide Precursor Material
}

\author{
Mingchun Jiao*, Jing Wang, Xianfang Yue, Yinping Zhou, Xiao Sun \\ Department of Physics and Information Engineering, Jining University, Qufu, China \\ Email: `jmch527@163.com, wangjingouc.126.com, xfyuejnu@jnxy.edu.cn, 1363339301@qq.com, 1983913233@qq.com
}

How to cite this paper: Jiao, M.C., Wang, J., Yue, X.F., Zhou, Y.P. and Sun, X. (2019) Preparation and Analysis of Porous Oxychloride Titanium Oxide Precursor Material. Journal of Applied Mathematics and Physics, 7, 1599-1605.

https://doi.org/10.4236/jamp.2019.78108

Received: July 14, 2019

Accepted: August 9, 2019

Published: August 12, 2019

\begin{abstract}
Oxychloride titanium oxide precursor material is one of the key substances produced by the giant electrorheological effect. In this paper, tetrabutyl titanate and oxalic acid and anhydrous ethanol are used to prepare porous titanyl oxalate precursor powder by precipitation method. The materials, morphology and grain size were analyzed by X-ray diffraction and electron microscope method. Conductivity was measured by a four-probe method, and then the infiltration property of water and silicone oil on the surface of the powder was investigated. The results show that Oxychloride titanium oxide precursor has good wettability to water and silicone oil. The wettability has little to do with the bath temperature and the $\mathrm{pH}$ of the solution. The contact angle of water on oxychloride titanium oxide precursor surface does not change greatly with the change of the bath temperature and $\mathrm{pH}$, ranging from $26.8^{\circ}$ to $31.1^{\circ}$. Similarly, the contact angle of silicone oil on the surface of the sample ranges from $9.5^{\circ}$ to $11.5^{\circ}$. So, silicone oil has better wettability on the surface of the sample than water. Porous titanium oxalate is an insulating material having a resistivity more than $1 \mathrm{G} \Omega \cdot \mathrm{cm}$. Oxychloride titanium oxide precursor is poor thermal stability, and it breaks down to generate $\mathrm{TiO}_{2}$ when the temperature is higher than $70^{\circ} \mathrm{C}$.
\end{abstract}

\section{Keywords}

Itanium Oxalate, Electrorheological Fluid, Coprecipitation

\section{Introduction}

Electrorheological fluid consists of a very high dielectric constant of solid particles distributed very uniformly in a low dielectric constant insulating oil [1]. In the absence of an applied electric field, it appears as a state of suspension. When 
an applied electric field is applied, it immediately changes from a liquid state to a solid state, producing a giant current effect, and its response speed is in the order of milliseconds. After losing the applied electric field, it changes from the solid state to the original state. Until recently, domestic and foreign research on electrorheological fluids has made new breakthroughs, and some high-performance new electrorheological fluids have been manufactured, making the application of electrorheological fluids strong.

Titanium oxalate precursor is one of the giant electro-rheological effect material components [2]. The earliest report of its electrorheological effect was the Lu Kunquan Research Group of the Institute of Physics Chinese Academy of Sciences. They synthesize an electrorheological fluid of calcium titanate system by co-precipitation of oxalic acid, which exhibits excellent performance and shear strength up to $100 \mathrm{KPa}$ [3]. This is an electrorheological fluid material with high application value. Later, there were many reports in succession [4] [5]. Titanium oxalate precursor, the precursor of titanyl oxalate exhibits a giant current effect, the mechanism cannot be explained by the dielectric model and the water bridge model and its mechanism can only be explained by the model of hyperpolarized water [6]. Water has two states in the precursor of titanyl oxalate, one is free water and the other is crystal water [7]. When an external electric field is applied by the electrorheological fluid, under the action of the external electric field, the dielectric particles are chained in the direction of the electric field under the action of the polarizing force [8]. At this time, the local field between the particles is as high as $10^{8}-10^{9} \mathrm{~V} / \mathrm{m}[6]$. Because the water molecules are polar molecule, and the local electric field between the media particles is not a uniform field, the free water molecules move from the high potential energy to the low potential energy. The water molecules between the media particles exist in a hyperpolarized state connected to the media particles [9]. If there is a small gap above the media particles, free water will move better inside and outside the media particles. This is conducive to the generation of giant current effects. We synthesized a porous titanium oxalate precursor and measured its physical properties such as density, particle size distribution, and electrical conductivity.

\section{Raw Materials and Experimental Methods}

Table 1 lists the most of chemical reagent required for this experiment. The main instruments and equipment used in the experiment of preparing samples

Table 1. Reagent used in experiment.

\begin{tabular}{ccccc}
\hline Reagent & Molecular & Relative molecular mass & Purity & Density $\left(20^{\circ} \mathrm{C}\right)$ \\
\hline Tetrabutyl titanate & $\mathrm{C}_{16} \mathrm{H}_{36} \mathrm{O}_{4} \mathrm{Ti}$ & 340.34 & $99.0 \%$ & $0.999 \mathrm{~g} / \mathrm{cm}^{3}$ \\
Absolute ethanol & $\mathrm{CH}_{3} \mathrm{CH}_{22} \mathrm{OH}$ & 46.07 & $99.7 \%$ & $0.789 \mathrm{~g} / \mathrm{cm}^{3}$ \\
Oxalic acid & $\mathrm{C}_{2} \mathrm{H}_{2} \mathrm{O}_{4} \cdot 2 \mathrm{H}_{2} \mathrm{O}$ & 126.07 & $99.5 \%$ & $1.653 \mathrm{~g} / \mathrm{cm}^{3}$ \\
Citric acid & $\mathrm{C}_{6} \mathrm{H}_{8} \mathrm{O}_{7} \cdot \mathrm{H}_{2} \mathrm{O}$ & 210.14 & $99.5 \%$ & - \\
Ammonia water & $\mathrm{NH}_{4} \mathrm{OH}$ & 35 & $26 \%$ & $0.91 \mathrm{~g} / \mathrm{cm}^{3}$ \\
\hline
\end{tabular}


are: electronic balance (Jingtian FA2004A type), measuring cup (250 ml), constant temperature water bath (HH8 type), constant temperature bidirectional magnetic heating stirrer (JB-3 type).

Instruments required for the experiment: electronic balance, measuring cup, constant temperature water bath, magnetic stirrer, mortar, beaker, etc.

The titanium oxalate precursor material was prepared by the literature coprecipitation method according to the experimental method in [7] [10]. First, $7.56 \mathrm{~g}$ of oxalic acid was weighed, and $20.56 \mathrm{~mL}$ of tetrabutyl titanate, $4.5 \mathrm{~mL}$ of water, and $100 \mathrm{~mL}$ of an anhydrous ethanol solution were weighed. After all the weighing was completed, the experiment was carried out in a constant temperature water bath at $30^{\circ} \mathrm{C}$, and tetrabutyl titanate was poured into vigorously stirred water to cause a hydrolysis reaction. After the completion of the hydrolysis, the solution was poured into an absolute ethanol solution and stirred for 10 minutes. Then, the oxalic acid was slowly poured into the vigorously stirred solution and stirred for 10 minutes to cause a polycondensation reaction to form a white precipitate. The solution was adjusted to $\mathrm{pH}=2$ with aqueous ammonia. The solution was then dried in a dry box at $50^{\circ} \mathrm{C}$ for $48 \mathrm{~h}$ and then dried at $120^{\circ} \mathrm{C}$ for 2 hour and then ground to form a white powder. Five samples were obtained at $\mathrm{pH}=2$ and temperatures of $30^{\circ} \mathrm{C}, 40^{\circ} \mathrm{C}, 50^{\circ} \mathrm{C}, 60^{\circ} \mathrm{C}$, and $70^{\circ} \mathrm{C}$, respectively.

The specific reaction equation is as follows [2]: First, the tetrabutyl titanate is hydrolyzed to form titanium hydroxide, and then the titanium hydroxide undergoes a polycondensation reaction to form polytitanium hydroxide. Finally, the polytitanium hydroxide is polycondensed with oxalic acid to form a titanyl oxalate precursor.

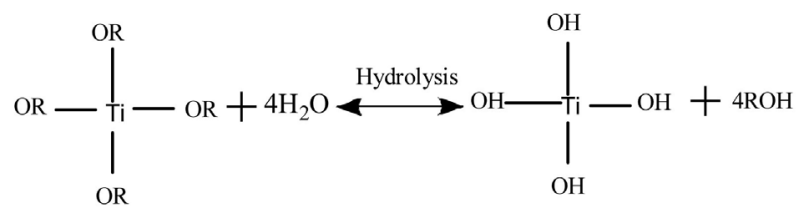

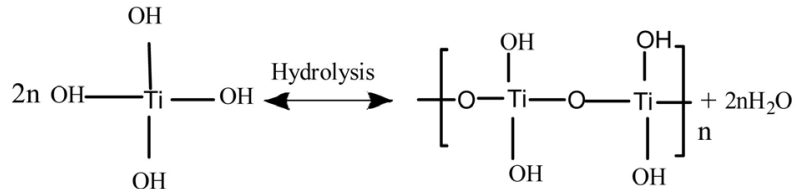

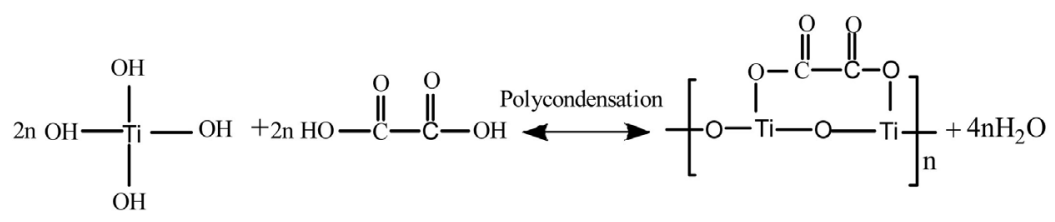

\section{Experimental Results and Analysis}

Figure 1 is a visual diagram of a sample obtained at $\mathrm{pH}=2$ and $\mathrm{T}=60^{\circ} \mathrm{C}$ of solution. As can be seen from Figure 1, the porous oxychloride titanium oxide precursor sample was white powder. The particles are even and fine. Among them, some particles have agglomeration. These samples and silicone oil are mixed in a certain ratio to prepare an electrorheological fluid. From this picture, 
the specific morphology and microstructure of the particles are not visible, so we performed an electron microscopy experiment to better observe the sample morphology.

Figure 2 is a scanning electron microscope image of the sample. From the figure, the phenomenon of particle agglomeration can be seen, and the distribution is relatively uniform. The surface of the particles is prominent, porous, not smooth, and the average particle size is micron-sized particles. Particle pores increase the specific surface area of the particles. Water molecules can be adsorbed in these holes. Under the action of the electric field, the particles are separated by charge due to polarization, the positive charge moves toward the end close to the negative electrode, and the negative charge moves toward the end close to the positive charge. Thus, a non-uniform electric field appears on the surface of the solid particles. Water molecules are polar molecules. In a non-uniform electric field, they are subjected to electric field forces and move to the surface of the particles to form a water bridge [11], but this time the water molecules are in a hyperpolarized state [10].

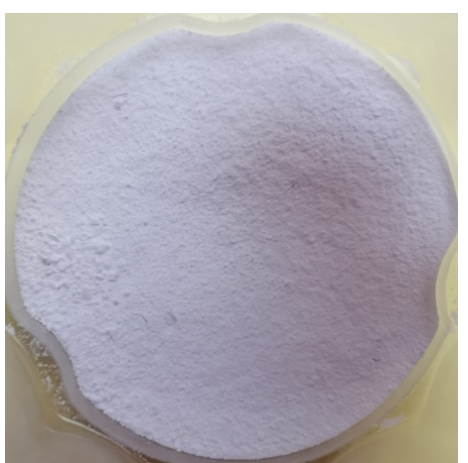

Figure 1. Sample photo of the sample prepared at $\mathrm{pH}=2, \mathrm{~T}=60^{\circ} \mathrm{C}$.

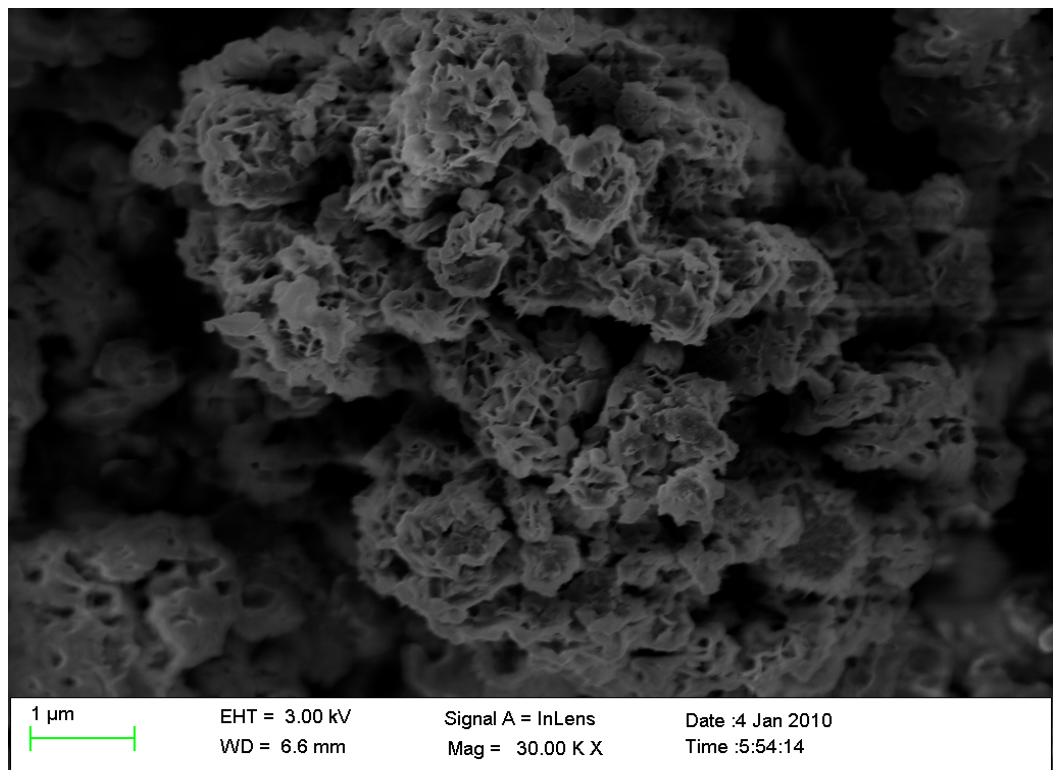

Figure 2. Scanning electron micrograph of the sample prepared at $\mathrm{pH}=2, \mathrm{~T}=60^{\circ} \mathrm{C}$. 
It can be seen from the three sets of picture pairs that the diffraction peak intensity of titanium oxalate is significantly higher than the diffraction peak intensity at $\mathrm{pH}=6$ at $\mathrm{pH}=2,4$ at the same temperature. It is indicated that as the amount of ammonia added increases, ions in the ammonia water also inhibit the crystallization of titanium oxalate. Or because the heating time is too long, the titanium oxalate loses the crystal water and then continues to decompose and form $\mathrm{TiO}_{2}$. In the figure, the type of $\mathrm{TiO}_{2}$ is brookite, which belongs to the orthorhombic system, and the crystal form is generally plate-like and blade-like (Figure 3).

\section{Invasive Analysis}

It can be seen from the data in Table 2 that the contact angle of water prepared at different temperatures is always between $29.1^{\circ}$ and $31.2^{\circ}$. It indicates that the reaction temperature has little effect on the wettability of the material. It can also be seen that the contact angle of silicone oil on the surface of the sample is between $9.5^{\circ}$ and $11.5^{\circ}$. The wettability of the silicone oil in the sample does not change with reaction temperature. This also shows that the generated materials are basically the same in the range of $30^{\circ} \mathrm{C}$ to $70^{\circ} \mathrm{C}$ of reaction temperature. However, the wetting property of silicone oil on the sample surface is greater than water.

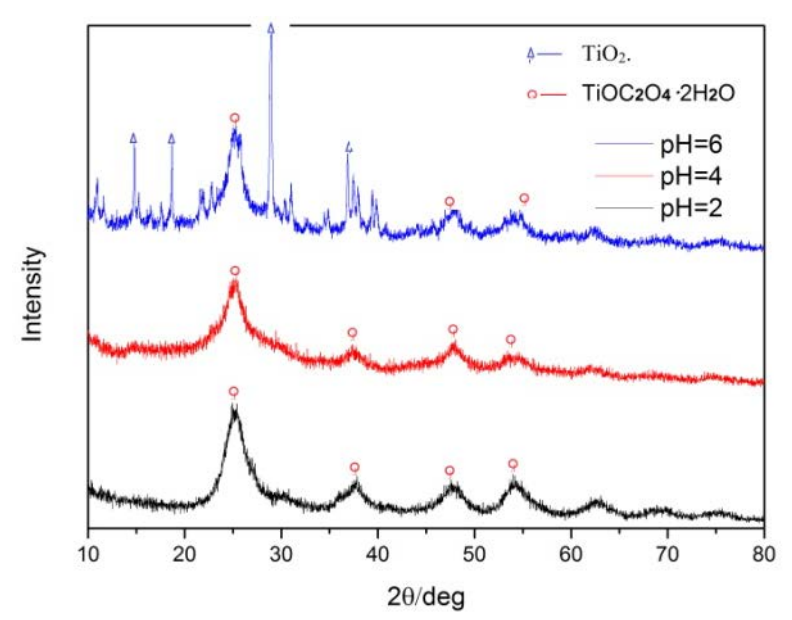

Figure 3. X-ray diffraction experiment of $\mathrm{TiOC}_{2} \mathrm{O}_{4} \cdot 2 \mathrm{H}_{2} \mathrm{O}$ at $\mathrm{pH}=2,4,6$ of solution.

Table 2. Surface contact angle of samples prepared at different temperatures and the $\mathrm{pH}$ $=2$.

\begin{tabular}{ccc}
\hline Temperature & Right contact angle $\left(^{\circ}\right)$ water & Right contact angle $\left(^{\circ}\right)$ silicone oil \\
\hline $30^{\circ} \mathrm{C}$ & 29.1 & 9.5 \\
$40^{\circ} \mathrm{C}$ & 31.1 & 11.5 \\
$50^{\circ} \mathrm{C}$ & 30.2 & 10 \\
$60^{\circ} \mathrm{C}$ & 30.1 & 10.5 \\
$70^{\circ} \mathrm{C}$ & 29.1 & 9.6 \\
\hline
\end{tabular}




\section{Four-Probe Method for Measuring Its Conductivity}

A good material for rheological fluids must be an insulator. We want to measure the resistivity of the material next. There are many ways to measure resistivity, and the four-probe method is a widely used standard method. First of all, the sample was pressed into a disk having a diameter of $1.5 \mathrm{~cm}$ and thickness of $1 \mathrm{~cm}$ by a laminator, and the resistivity of the sample was measured by four-probe method. The measured resistivity was greater than $1 \mathrm{G} \Omega \cdot \mathrm{cm}$. It indicates that porous oxychloride titanium oxide precursor Material is good insulator.

\section{Sum Up}

Porous titanium oxalate is an insulating material having a resistivity more than 1 $\mathrm{G} \Omega \cdot \mathrm{cm}$. The co-precipitation method has poor thermal stability of titanium oxalate. In the process of preparation, the drying time is too long, so that the titanium oxalate loses crystal water and then continues to decompose and form $\mathrm{TiO}_{2}$. $\mathrm{Ti}$ tanium oxalate is material that has good wettability to both water and silicone oils. The wetting angle of water on titanium oxalate is between $26.8^{\circ}$ and $3.11^{\circ}$. The contact angle of silicone oil on the surface of the sample is between $9.5^{\circ}$ $11.5^{\circ}$. The infiltration of silicone oil on the surface of the sample is better than the wettability of water.

\section{Acknowledgements}

This work was supported by the National Science Foundation of China (Grant Nos. 11347176 and 11447014) and the Innovative Experimental Project of Jining University (Grant No. cx201883).

\section{Conflicts of Interest}

The authors declare no conflicts of interest regarding the publication of this paper.

\section{References}

[1] Hao, T. (2001) Electrorheological Fluids. Adv. Mater, 13, 1847-1857. https://doi.org/10.1002/1521-4095(200112)13:24<1847::AID-ADMA1847>3.0.CO;2A

[2] Yang, S.H., Zhao, L.S., Wang, Q., et al. (2013) Composition Analysis and Mechanism Approach of the Electrorheological fluids Based on the Precursor of $\mathrm{TO}_{2}$. Acta Phys. Sin, 62, 164701.

[3] Lu, K.Q., Shen, R., Wang, X.Z., et al. (2007) Polarmolecule Type Electrorheological Fluids. Physics, 36, 742-749. https://doi.org/10.1142/9789812771209_0011

[4] Xu, L., Tian, W.J., Wu, X.F., et al. (2008) Polar-Molecules-Driven Enhanced Colloidal Electrostatic Interactions and Their Applications in Achieving High active Electrorheological Materials. J. Mater. Res, 23, 409-417. https://doi.org/10.1557/JMR.2008.0057

[5] Dong, X.F., Luo, X.W., Zhao, H., et al. (2013) Effects of Terminal Group of Silicone Oil on Properties of Oxalate Group-Modified Amorphous Titanium Oxide Nano- 
particles-Based Electrorheological Fluids. Journal of Functional Materials, 44, 2697-2670.

[6] Jiao, M.C., Sun, G., Wang, Q., et al. (2012) Multiple Scattering of Approaches on the Elctrorheological Fluids. Mod. Phys. Lett. B, 26, Article ID: 1150007.

https://doi.org/10.1142/S0217984911500072

[7] Boudaren, C., Bataille, T., Auffrédic, J.P., et al. (2003) Synthesis, Structure Determination from Powder Diffraction Data and Thermal Behaviour Of Titanium(IV) Oxalate $\left[\mathrm{Ti}_{2} \mathrm{O}_{3}\left(\mathrm{H}_{2} \mathrm{O}\right)_{2}\right]\left(\mathrm{C}_{2} \mathrm{O}_{4}\right) \cdot \mathrm{H}_{2} \mathrm{O}$. Solid State Sci, 5, 175-182. https://doi.org/10.1016/S1293-2558(02)00091-2

[8] Shen, M., Cao, J.G. and Xue, H.T. (2006) Structure of Polydisperse Electrorheological Fluids: Experiment and Theory. Chem. Phys. Lett, 423, 165-169. https://doi.org/10.1016/j.cplett.2006.03.066

[9] Choi, Y.C., Pak, C. and Kim, K.S. (2006) Electric Field Effects on Water Clusters (n = 3 - 5): Systematic Ab Initio Study of Structures, Energetics, and Transition States. J. Chem. Phys, 124, Article ID: 094308. https://doi.org/10.1063/1.2173259

[10] Jiao, M.C., Tan, W. and Yue, X.F. (2012) Morphology Study of Calcium Titanate Precursor Prepared by Sol-Gel Method. J. Jining University, 37, 5-8.

[11] See, H., Tamura, H. and Doi, M. (1993) The Role of Water Capillary Forces in Electro-Rheological Fluids. J. Phys. D, 26, 746-752.

https://doi.org/10.1088/0022-3727/26/5/005 